\title{
Improving the diagnostic quality and adequacy of shoulder radiographs in a District General Hospital
}

To cite: Richards B, Riley J, Saithna A. Improving the diagnostic quality and adequacy of shoulder radiographs in a District General Hospital. BMJ Quality Improvement Reports 2016;5:u209855.w3501. doi:10.1136/bmjquality. u209855.w3501

Received 2 May 2016 Revised 18 July 2016 Published Online First 4 August 2016

Southport and Ormskirk NHS Hospitals Trust

Correspondence to Bethany Richards beth. richards@doctors.org.uk

\section{ABSTRACT}

A high rate of suboptimal shoulder radiographs was identified during a service evaluation exercise in our orthopaedic outpatient clinics. Inadequate radiographs require a return to the radiology department for further imaging, a resultant increased workload, delays in the clinic, increased radiation for patients, and inconvenience and decreased patient satisfaction. Furthermore, if a sub-optimal radiograph is accepted there is concern that diagnoses may be missed. The aim of this project was to decrease the rate of suboptimal radiographs by delivering a teaching package directed towards quality improvement.

Evaluation criteria were set for standard orthopaedic shoulder radiographs (Anterior-posterior, axillary, and Velpeau views). Baseline data collection was performed over three, two-week periods and included all patients attending the shoulder clinic. The percentage of x-rays which were deemed adequate was only $19.4 \%$ for anterior-posterior views and $57.9 \%$ for axillary views.

A comprehensive educational package was delivered to radiographers. This included a formal PowerPoint based teaching session, hands on training with practice using a skeleton, posters with step-by step instructions on how to obtain an adequate image, and PDF aide memoires suitable for viewing on a smartphone. Two subsequent two-week periods of data collection were performed to evaluate the benefit of this intervention.

Delivery of focussed training and provision of easily accessible aide memoires to facilitate improved quality of radiographs resulted in a significant $(p<0.05)$ reduction in the rate of inadequate images. There was also a significant decreases in the rate of return to the radiology department for repeat imaging.

\section{PROBLEM}

Radiographs are often the first investigation utilised by specialist orthopaedic shoulder surgeons in the assessment of their patients in both the emergency department and outpatient clinics. ${ }^{1}$ They allow visualisation of the complex anatomy of the shoulder girdle and are essential for the accurate diagnosis and safe assessment of patients. ${ }^{2}$ It was noticed during the outpatient clinics of one specialist shoulder consultant at Southport and Ormskirk Hospitals NHS Trust, UK, that shoulder radiographs were sometimes suboptimal, particularly anterior-posterior (AP) and axillary views. These clinics consist of three sessions per week, one at Ormskirk Hospital and two at Southport Hospital. They are elective orthopaedic and fracture clinics which cover between 15-30 patients per clinic. Therefore, if these views are inadequate for diagnosis, and patients need to be returned to the radiology department to have repeat imaging there is significant delay in the clinic and an increased workload for the radiology department. This is also inconvenient for patients and furthermore, if a sub-optimal radiograph is accepted or not recognised, and the patient is not sent back for repeat imaging, diagnoses may be given incorrectly or even missed.

\section{BACKGROUND}

The most commonly used radiographic views are the AP and axillary views. AP views are used to visualise a multitude of shoulder pathologies including gleno-humeral osteoarthritis and humeral neck or shaft fractures. ${ }^{3}$ An ideal AP image is deemed to have no overlap between the glenoid fossa and the humeral head, the glenoid fossa has a small width but a large height and it's anterior and posterior rims are superimposed (Attachment 1 - Figure 1$).{ }^{4}$ It is often difficult to achieve these features and as such, standard AP views of the shoulder frequently show a large amount of overlap making joint space evaluation impossible and thus ensuring the diagnosis of osteoarthritis is difficult to make. ${ }^{3}$

Recent research has focussed upon the most accurate methods of obtaining satisfactory AP radiographs. A study by Braunstein et al. advocated the use of a 'Fulcrum view'. This is based upon cadaver research showing that the line between the anterior coracoid process tip and the postero-lateral angle of the acromion is very closely related to the 


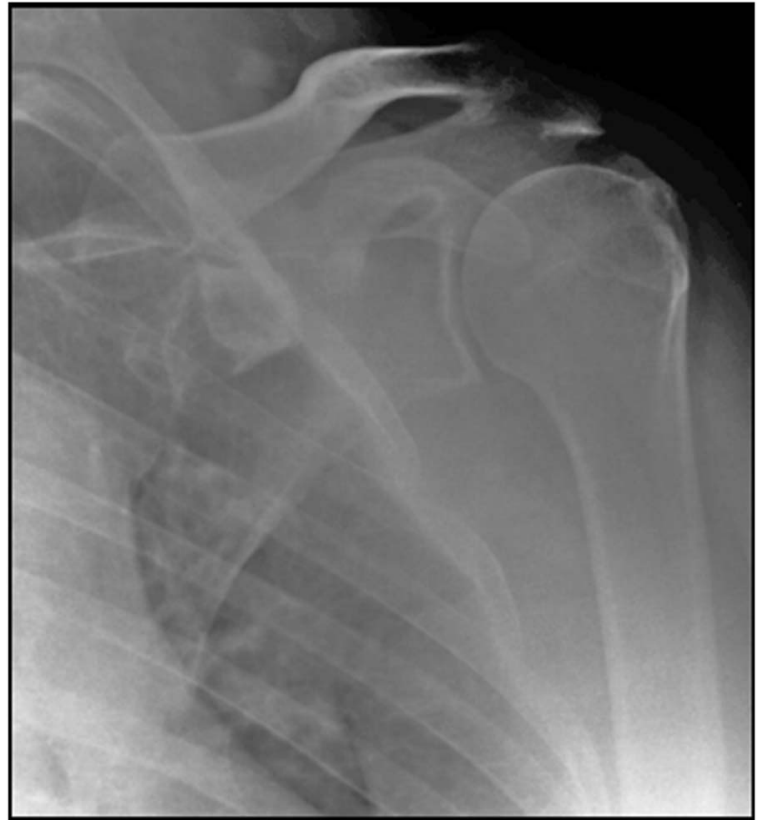

Figure 1 An adequate AP view meeting the evaluation criteria. Of particular note is the clear joint space between the glenoid fossa and the humeral head.

plane of the gleno-humeral joint, in fact, they deviate by only 1.8 degrees. ${ }^{4}$ Once the above landmarks have been identified, the cassette is placed at 90 degrees to the line established and the x-ray beam is centred on the tip of the coracoid process with a 10 degree caudal tilt (Attachment 1 - Figure 2). ${ }^{4}$ This 'Fulcrum view' has been shown to produce ideal AP radiographs which meet the criteria outlined previously. It also suggests that the overlap frequently seen on inadequate AP images is due to the lack of reliance on the superficial anatomical landmarks used to establish the joint line thus meaning the radiograph is not taken in the plane of the joint. ${ }^{4}$

Axillary views are also frequently performed inadequately. The purpose of this view is for the requesting healthcare professional to assess the relationship between the humeral head and the glenoid fossa and to visualise lesions to the humeral head, lesser tubercle, and glenoid rim. ${ }^{2}{ }^{3}$ Therefore, it is often used if the patient has a potential dislocation or may be requested to assess the damage done by recurrent dislocations. ${ }^{2}$ It also allows evaluation of the gleno-humeral joint from below. ${ }^{2}$ In these radiographs, an adequate film must have a clear view of the joint space between the glenoid fossa and the humeral head, the superior and inferior edges of the glenoid fossa should be superimposed, and there should be a clear view of other anatomy such as the coracoid process and the acromioclavicular joint (ACJ) visible through the humeral head (Attachment 1 - Figure 3). ${ }^{56}$ Unfortunately, a 90 degree abduction angle of the shoulder is required for this view and this is often uncomfortable or even extremely painful for those patients who have sustained a shoulder injury and thus axillary radiographs are often performed inadequately. ${ }^{3}$

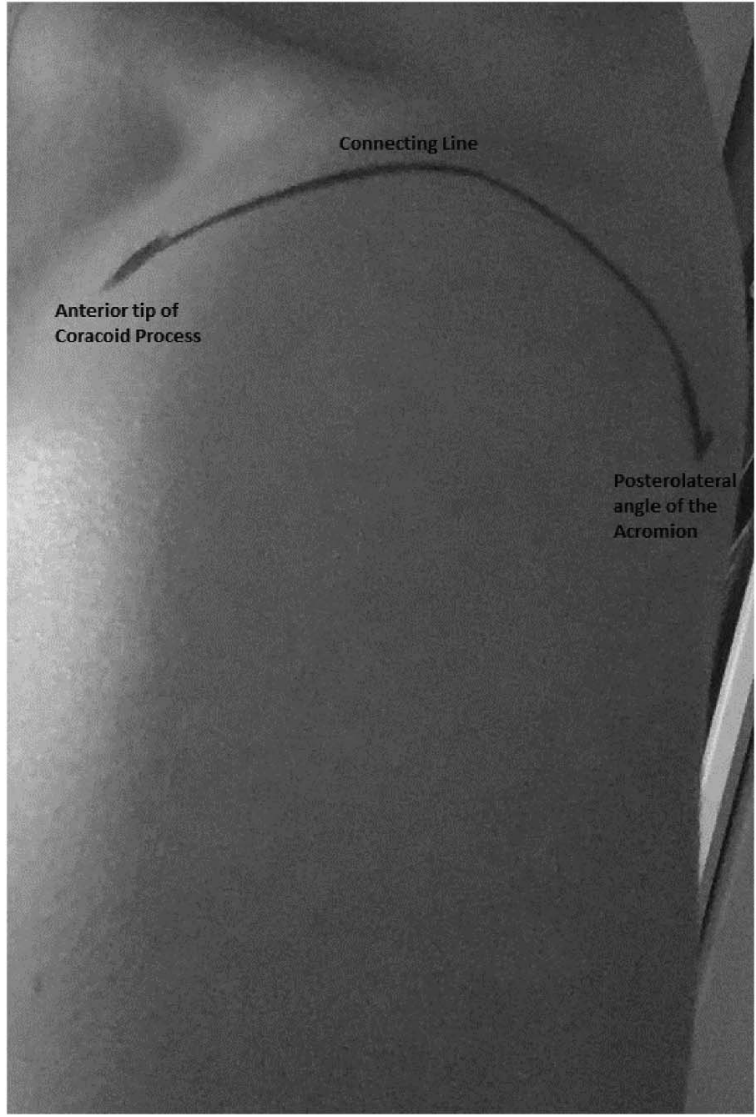

Figure 2 Photograph to show surface anatomy identified by Braunstein et al. Note the anterior tip of the corocoid process, the posterolateral angle of the acromian and the connecting line which runs between them in the plane of the glenohumeral joint.

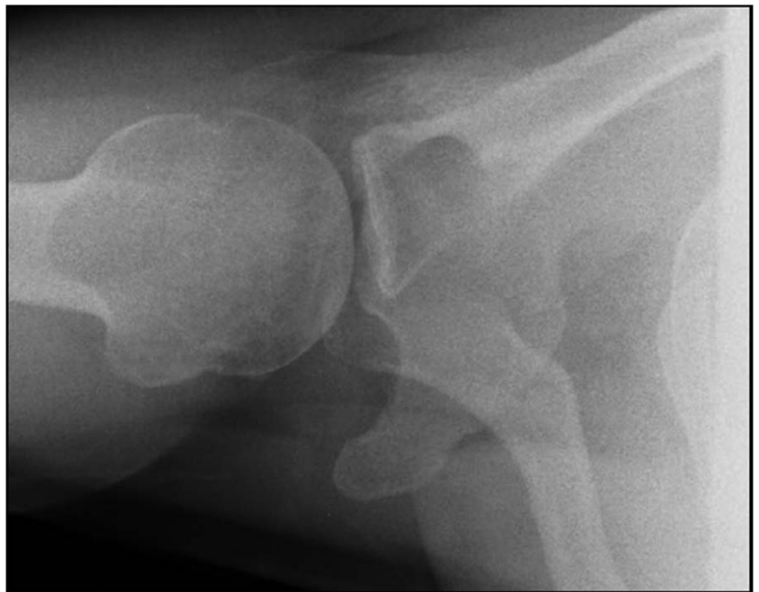

Figure 3 An adequate axillary view as per the evaluation criteria. Again, note the unobscured joint space between the humeral head and the glenoid fossa.

In the trauma patients mentioned above, an alternative axillary view, known as the Velpeau view, can be used and therefore reduces the need to cause the patient any pain. ${ }^{7}$ It does this by allowing the patient to remain in the sling and does not require any shoulder 
abduction. In these radiographs it is expected to be able see less than a standard axillary view but an adequate attempt would allow visualisation of the anterior and posterior aspects of both the humeral head and the glenoid fossa. ${ }^{7}$

\section{BASELINE MEASUREMENT}

In order to deem a shoulder radiograph adequate a number of evaluation criteria were created for each view using the background information gathered above and also following discussion with an orthopaedic surgeon with a specialist interest in shoulder surgery. They are outlined in attachment 2.

For baseline data collection, shoulder radiographs were assessed over a total of nine clinics across three two-week periods. All patients requiring a shoulder radiograph were included in the baseline measurement $(n=72)$. It was recorded which views patients were subject to, whether they were adequate or inadequate and whether they were sent back for repeat imaging following an inadequate $\mathrm{x}$-ray.

This data collection confirmed a large number of inadequate x-rays. Of 72 patients, 36 required an AP view and of those only $19.4 \%$ were adequate. 19 patients required an axillary view radiograph and only $57.9 \%$ were adequate. However, only one patient required a trauma axillary view and this was done adequately. Two patients were sent for repeat x-rays, one required a repeat of an $\mathrm{AP}$ and the other a repeat of both an AP and axillary view. Therefore, of $36 \mathrm{AP}$ views carried out $5.5 \%$ were repeated and of the 19 axillary views $5.2 \%$ were repeated (see supplementary file "Evaluation Criteria").

\section{DESIGN}

After analysing the results from the baseline data collection, our team, consisting of a specialist shoulder orthopaedic surgeon (the senior author), a core surgical trainee, a senior radiographer and a fifth year medical student devised a SMART aim. This was to improve radiographic imaging of the shoulder at three busy orthopaedic outpatient clinics held across Southport and Ormskirk Hospitals NHS Trust. Using the Fulcrum technique for AP views, the Braunstein et al trial improved their number of adequate radiographs to $64 .^{4}$ We hoped to match this achievement and improve our baseline adequate percentages of $19.4 \%$ (AP) and $57.9 \%$ (Axillary) to at least $64 \%$ over six months. An intervention in the form of an educational package to be carried out in two one hour sessions (one at each hospital site where the outpatient clinics occur) was created following a discussion between the senior author, the lead radiographer, and a number of radiographers about what they considered to be adequate radiographs. It was recognised that radiographers were unaware what criteria determined an adequate radiograph thus highlighting the need for education.

The above intervention was felt to be sustainable due to its projected low cost, the teaching material being freely available to all radiographers and the ability to repeat, alter, or extend future teaching sessions in response to feedback.

\section{STRATEGY}

Improvement Cycle 1

Following the identification of the problem and baseline data collection, our aim was to improve the adequacy of shoulder x-rays at Southport and Ormskirk NHS trust. Specifically we aimed for over $64 \%$ of shoulder x-rays to be adequate.

An education package was developed and included posters for display throughout the department which highlighted the evaluation criteria for each radiographic view of the shoulder (AP, axillary, and Velpeau) and step-by-step instructions to producing an adequate image. In addition to this we carried out a teaching session consisting of formal PowerPoint aided instruction and a practical session using a model skeleton. This practical session was particularly important as it allowed direct visualisation of the anatomy of the shoulder girdle which is imperative to good visualisation of the glenohumeral joint space. The radiographers were able to easily identify the coracoid process and posterolateral corner of the acromion on the skeleton and thus could transfer this knowledge to patients and position them as outlined in the Braunstein et al study and successfully carry out the Fulcrum view. This session was carried out across both hospital sites to ensure any radiographer likely to perform shoulder x-rays were aware of the intervention. Time was also provided for radiography staff to ask any questions regarding the audit and also for discussion between them and the orthopaedic department.

Data collection two weeks following the introduction of this intervention showed a dramatic improvement, exceeding the aims set by the team. However, radiographers stated that when carrying out shoulder x-rays the posters weren't always readily available. This was taken on board and a second improvement cycle carried out.

Improvement Cycle 2

The aims of this cycle were to maintain or exceed previous results and demonstrate sustainability of the improvement.

Following the radiographer's feedback, PDF posters were provided which they could access at any time on their mobile phones. PDF checklists outlining pre- and post-radiograph checks were also provided as aide memoires to help increase improvement sustainability.

A final data collection three months following the introduction of these resource confirmed the improvement was sustainable, at least in the short term.

In addition, discussion between the trust audit and orthopaedic department has generated a future action plan which aims to improve the adequacy of shoulder x-rays at the clinics of other orthopaedic consultants within the trust by introducing these views as the default shoulder views for all radiographs requested by the orthopaedic department. 
A detailed overview of these cycles can be seen in supplementary file 1 .

\section{RESULTS}

The second data collection, two weeks following the intervention of the educational package, confirmed a statistically significant increase in the number of adequate $\mathrm{x}$-rays for all three views. The target of $64 \%$ was exceeded with $93.3 \%$ of the 15 AP views carried out being adequate (Chi-Square Statistic: 23.8661. $\mathrm{P}<0.01$ ) and $92.3 \%$ of the 13 axillary views being adequate (Chi-Square Statistic: 4.1462. $\mathrm{P}=0.042$ ). The percentage of repeat x-rays was reduced from $5.5 \%$ and $5.2 \%$ for AP and axillary views respectively to $0 \%$ across all views.

The third data collection, three months following the intervention, confirmed the change was sustainable. The $64 \%$ target was again exceeded with $80 \%$ adequacy of the 15 AP views taken (Chi-Square statistic: 16.61. $\mathrm{P}<0.01)$ and $93.3 \%$ adequacy of the 15 axillary views taken (Chi-Square Statistic: 10.4429. The $\mathrm{P}<0.01$ ). The percentage of repeated x-rays remained at $0 \%$. Furthermore, as aforementioned, discussion sparked following the second improvement cycle has generated a future action plan for the project which involves using the strategy to improve shoulder radiograph imaging across the clinics of the other consultant orthopaedic surgeons within Southport and Ormskirk Hospitals NHS Trust (see supplementary file "Run Chart").

\section{LESSONS AND LIMITATIONS}

There are many important lessons to take from completing this quality improvement project. First of all, ensuring you have the right people on board; in this case, we needed to engage our main stakeholders, the radiographers. It is important that they understand the benefits to the hospital and the patients. To achieve this it was especially important to deliver the teaching session in such a way as to reassure them that you are not being 'picky' or undermining them in any way. We received positive feedback from the radiographers that we had achieved this and that they felt they could undertake meaningful discussion with us during the sessions. It was also imperative to ensure that the maximum number of radiographers were reached and this encompasses one of the limitations of the project. Carrying out only one teaching session at each hospital site inevitably meant we would not be able to engage every radiographer due to shift patterns. We attempted to combat this by producing simple, easy to read posters and PDFs which the non-attending staff could access. We also left contact details with a designated member of the radiography team at each hospital site so we could be contacted if any queries arose. Aside from these actions, we relied on word of mouth throughout the department.

A criticism regarding our educational package is that it consists of a number of small interventions. We therefore cannot be sure which had the largest impact on the observed improvement; the posters, the teaching, the practical demonstration, or the introduced resources of PDF posters and checklists. By trialling each part of the education package on small groups of radiographers, and analysing the improvement data from each group, we could identify the intervention which has the most impact. In addition, if we were to have built up our education package in this way we would also have been able to use Plan, Do, Study, Act (PDSA) Cycles as opposed to our improvement cycles. This is because PDSA cycles allow the user to make smaller changes over a rapid time scale in contrast to our cycles which made larger changes over a longer period of time.

A further limitation is that data collection was only carried out in one surgeon's outpatient clinic. In order to assess the external validity of the results, it would be useful to analyse the adequacy of shoulder x-rays in a second shoulder specialist clinic or even in the emergency department. Following on from this, future projects could obtain input from other hospital trusts to even further test the generalisability of the data. In addition to this, it would be beneficial to carry out a further data collection for this project, our current plan is to do this at 12 months to observe long term sustainability as our short term follow up appears to be sustainable.

\section{CONCLUSION}

A significant problem regarding the adequacy of shoulder x-rays in a district general hospital was identified with only $19.4 \%$ of AP views and $57.9 \%$ of axillary views allowing for proper evaluation and diagnosis of patients presenting to busy orthopaedic outpatient clinics. This meant patients were being sent for repeat imaging causing unnecessary delay in clinics for patients and producing extra cost for the hospital. An education package was put together focussing on teaching radiographers how to produce the perfect image. This centred on the Fulcrum view as described by Braunstein et al plus evaluation criteria and step-by-step posters and PDFs for all three views. A further data collection was carried out following this intervention with the target for adequate $\mathrm{x}$-rays set at $64 \%$ as dictated by the improvement results of the Braunstein et al. trial when they introduced the Fulcrum view. It was found that this target was exceeded, $93.3 \%$ of AP views and $92.3 \%$ of axillary views were regarded as adequate. Further to this, a third data collection three months following the introduction of mobile phone PDF posters and checklists shows the permanence of the change with adequacy remaining above target; $80 \%$ for AP views and $93.3 \%$ for axillary views. In addition, discussion following the second improvement cycle between the audit and orthopaedic department has generated a future action plan which aims to improve the adequacy of shoulder x-rays at the clinics of other orthopaedic consultants within the trust.

Acknowledgements Mr Yasen

Declaration of interests Nothing to declare 
Ethical approval This project was not subject to ethical review.

Open Access This is an open-access article distributed under the terms of the Creative Commons Attribution Non-commercial License, which permits use, distribution, and reproduction in any medium, provided the original work is properly cited, the use is non commercial and is otherwise in compliance with the license. See:

- http://creativecommons.org/licenses/by-nc/2.0/

- http://creativecommons.org/licenses/by-nc/2.0/legalcode

\section{REFERENCES}

1. Sandstrom CK, Kennedy SA, Gross JA. Acute shoulder trauma: what the surgeon wants to know. Radiographics 2015;

Mar-Apr;35:475-92
2. Sanders TG, Jersey SL. Conventional radiography of the shoulder. Semin Roentgenol 2005;40:207-22.

3. Greenspan A. Orthopedic Imaging: A Practical Approach. 6th ed. LWW, 2014

4. Braunstein V, Kirchhoff C, Ockert B, Sprecher CM, Korner M, Mutschler W, et al. Use of the fulcrum axis improves the accuracy of true anteroposterior radiographs of the shoulder. J Bone Joint Surg Br. 2009;91-B:1049-53

5. Frank E, Long B, Smith B, Merrill V. Merrill's Atlas of Radiographic Positioning \& Procedures. 12th ed. Moseby, 2011

6. Ahmad N. X-Ray Patient Positioning Manual. 2008. Available at: http://cdn.auntminnie.com/user/documents/content_documents/ X-Ray_Patient_Positioning_Manual_080402.pdf

7. Lee JH. Proposal of Modified Velpeau View as an Alternative Test Method of Velpeau View and the Visual Comparison. European Society of Radiology, EPOS. DOI: 10.1594/ecr2013/C-0504 